Article

\title{
On Solutions to the Set-Theoretical Yang-Baxter Equation in Wajsberg-Algebras
}

\author{
Tahsin Oner * (iD) and Tugce Katican \\ Department of Mathematics, Ege University, 35100 Izmir, Turkey; tugcektcn@gmail.com \\ * Correspondence: tahsin.oner@ege.edu.tr
}

Received: 26 October 2017; Accepted: 17 January 2018; Published: 20 January 2018

\begin{abstract}
In this work, we introduce Wajsberg algebras which are equivalent structures to MV-algebras in their implicational version, and then we define new notions and give new solutions to the set-theoretical Yang-Baxter equation by using Wajsberg algebras.
\end{abstract}

Keywords: Yang-Baxter equation; Wajsberg-algebra

\section{Introduction}

The Yang-Baxter equation which was initially used in theoretical physics [1] and statical mechanics [2-4] has gradually attracted the attention of researchers from various areas of science. In particular, this equation is considered in areas such as link invariant, $C^{*}-$ algebras, conformal field theory, quantum computing, quantum groups, quantum mechanics, knot theory, intregrable systems, non-commutative geometry, etc. (see, for example, [5-11]).

The problem to find and study (set-theoretical) solutions of the Yang-Baxter equation has attracted many authors.

The Yang-Baxter equation involves a linear operator $R: V \otimes V \longrightarrow V \otimes V$, where $V$ is a vector space and has the form

$$
R^{12} \circ R^{23} \circ R^{12}=R^{23} \circ R^{12} \circ R^{23} \text { in } \operatorname{End}(V \otimes V \otimes V)
$$

where $1 \leq n, m \leq 3$ and $R^{n m}$ means $R$ acting in the $n$-th and $m$-th components. In the last years, many set-theoretical solutions of this equation have given rise to the connection with various mathematical structures, such as quantum binomial algebras [12,13], semigroups of I-type and Bieberbach groups [14,15], bijective 1-cocyles [16], semisimple minimal triangular Hopf algebras [17], dynamical systems [18], and geometric crystals [19].

Since Wajsberg has shown that $\infty$-valued Lukasiewicz logics were complete with respect to the axioms postulated by Lukasiewicz, these logics were shown to play an important role in the study of quantum physics. We then wish to investigate the Yang-Baxter equation rather in relation with Wajsberg-algebras than quantum physics.

The set-theoretical solutions to the Yang-Baxter equation using MV-algeras were given by [11].

In this paper, we give some solutions to the set-theoretical Yang-Baxter equation in Wajsberg algebras.

\section{Preliminaries}

In this section, we present some definitions and properties of Wajsberg-algebras.

Definition 1. [20] A Wajsberg algebra (briefly, a W-algebra) is a structure $\langle A, \longrightarrow, \neg, 1\rangle$ satisfying the following equations, where $A$ is a nonempty set, $\neg$ is a unary operation on $A, \longrightarrow$ is a binary operation on $A$, and 1 is a distinguished element of $A$ : 
$(W 2) \quad(a \longrightarrow b) \longrightarrow((b \longrightarrow c) \longrightarrow(a \longrightarrow c))=1$

(W3) $\quad(a \longrightarrow b) \longrightarrow b=(b \longrightarrow a) \longrightarrow a$

(W4) $\quad(\neg a \longrightarrow \neg b) \longrightarrow(b \longrightarrow a)=1$.

Moreover, bounded commutative BCI/BCK-algebras (BCK-algebras are special cases of BCI-algebras, for example, see [21]), in their implicational notation, are known as Wajsberg algebras (see also [22]).

Lemma 1. [20] Let $\langle A, \longrightarrow, 1\rangle$ be a system satisfying (W1), (W2) and (W3). Then the following properties hold for every $a, b$ and $c$ in $A$ :

(W5) $\quad a \longrightarrow a=1$

(W6) If $a \longrightarrow b=b \longrightarrow a=1$, then $a=b$.

(W7) $\quad a \longrightarrow 1=1$

(W8) $\quad a \longrightarrow(b \longrightarrow a)=1$

(W9) If $a \longrightarrow b=b \longrightarrow c=1$, then $a \longrightarrow c=1$.

$(W 10) \quad(a \longrightarrow b) \longrightarrow((c \longrightarrow a) \longrightarrow(c \longrightarrow b))=1$

(W11) $\quad a \longrightarrow(b \longrightarrow c)=b \longrightarrow(a \longrightarrow c)$.

Lemma 2. [20] The following equations hold in every W-algebra:

$$
\begin{aligned}
\text { (i) } & \neg 1 \longrightarrow a=1, \\
\text { (ii) } & \neg a=a \longrightarrow \neg 1, \\
\text { (iii) } & \neg \neg a=a, \\
\text { (iv) } & a \longrightarrow b=\neg b \longrightarrow \neg a .
\end{aligned}
$$

\section{Solutions to the Yang-Baxter Equation in W-Algebras}

In this section, we provide solutions to the set-theoretical Yang-Baxter equation in W-algebras. Let $V$ be a vector space over a field $F$. We denote by $\tau: V \otimes V \longrightarrow V \otimes V$ the twist map defined by $\tau(v \otimes w)=w \otimes v$ and by $I: V \longrightarrow V$ the identity map over the space $V$; for a F-linear map $R: V \otimes V \longrightarrow V \otimes V$, let $R^{12}=R \otimes I, R^{23}=I \otimes R$, and $R^{13}=(I \otimes \tau)(R \otimes I)(\tau \otimes I)$.

Definition 2. [10] A Yang-Baxter operator is an invertible F-linear map $R: V \otimes V \longrightarrow V \otimes V$, and it satisfies the braid condition (also called the Yang-Baxter equation):

$$
R^{12} \circ R^{23} \circ R^{12}=R^{23} \circ R^{12} \circ R^{23} .
$$

If $R$ satisfies Equation (1), then both $R \circ \tau$ and $\tau \circ R$ satisfy the quantum Yang-Baxter equation:

$$
R^{12} \circ R^{13} \circ R^{23}=R^{23} \circ R^{13} \circ R^{12} .
$$

(QYBE)

The following definition enables us to constitute a relationship between the set-theoretical Yang-Baxter equation and $\mathrm{W}$-algebras.

Definition 3. [10] Let $X$ be a set and $S: X^{2} \longrightarrow X^{2}, S\left(p_{1}, p_{2}\right)=\left(p_{1}^{\prime}, p_{2}^{\prime}\right)$ be a map. The map $S$ is a solution to the set-theoretical Yang-Baxter equation if it satisfies the following identity:

$$
S^{12} \circ S^{23} \circ S^{12}=S^{23} \circ S^{12} \circ S^{23},
$$

where

$$
S^{12}: X^{3} \longrightarrow X^{3}, \quad S^{12}\left(p_{1}, p_{2}, p_{3}\right)=\left(p_{1}^{\prime}, p_{2}^{\prime}, p_{3}\right),
$$




$$
\begin{aligned}
& S^{23}: X^{3} \longrightarrow X^{3}, \quad S^{23}\left(p_{1}, p_{2}, p_{3}\right)=\left(p_{1}, p_{2}^{\prime}, p_{3}^{\prime}\right), \\
& S^{13}: X^{3} \longrightarrow X^{3}, \quad S^{13}\left(p_{1}, p_{2}, p_{3}\right)=\left(p_{1}^{\prime}, p_{2}, p_{3}^{\prime}\right) .
\end{aligned}
$$

Now, we provide solutions to the set-theoretical Yang-Baxter equation by using W-algebras.

Theorem 1. Let $\langle A, \longrightarrow, \neg, 1\rangle$ be a W-algebra. Then $S(a, b)=(\neg a \longrightarrow b, \neg 1)$ is a solution to the set-theoretical Yang-Baxter equation.

Proof. $S^{12}$ and $S^{23}$ are defined in the following forms:

$$
S^{12}(a, b, c)=(\neg a \longrightarrow b, \neg 1, c), \quad S^{23}(a, b, c)=(a, \neg b \longrightarrow c, \neg 1) .
$$

For all $(a, b, c) \in A^{3}$, we get

$$
\begin{aligned}
\left(S^{12} \circ S^{23} \circ S^{12}\right)(a, b, c) & =\left(S^{12} \circ S^{23}\right)\left(S^{12}(a, b, c)\right) & & \\
& =\left(S^{12} \circ S^{23}\right)(\neg a \longrightarrow b, \neg 1, c) & & \\
& =S^{12}\left(S^{23}(\neg a \longrightarrow b, \neg 1, c)\right) & & \\
& =S^{12}(\neg a \longrightarrow b, \neg \neg 1 \longrightarrow c, \neg 1) & & \\
& =S^{12}(\neg a \longrightarrow b, 1 \longrightarrow c, \neg 1) & & \text { (Lemma 2 (iii)) } \\
& =S^{12}(\neg a \longrightarrow b, c, \neg 1) & & \text { (W1) } \\
& =(\neg(\neg a \longrightarrow b) \longrightarrow c, \neg 1, \neg 1) & & \text { (Lemma 2 (iii)-(iv)) } \\
& =(\neg c \longrightarrow(\neg b \longrightarrow a), \neg 1, \neg 1) & & \text { (W11) } \\
& =(\neg b \longrightarrow(\neg c \longrightarrow a), \neg 1, \neg 1) & & \text { (Lemma 2 (iii)-(iv)) } \\
& =(\neg b \longrightarrow(\neg a \longrightarrow c), \neg 1, \neg 1) & & \text { (W11) } \\
& =(\neg a \longrightarrow(\neg b \longrightarrow c), \neg 1, \neg 1) & & ((\text { W1) and Lemma 2 (iii)) } \\
& =(\neg a \longrightarrow(\neg b \longrightarrow c), \neg \neg 1 \longrightarrow \neg 1, \neg 1) & & \\
& =S^{23}(\neg a \longrightarrow(\neg b \longrightarrow c), \neg 1, \neg 1) & & \\
& =S^{23}\left(S^{12}(a, \neg b \longrightarrow c, \neg 1)\right) & & \\
& =\left(S^{23} \circ S^{12}\right)(a, \neg b \longrightarrow c, \neg 1) & & \\
& =\left(S^{23} \circ S^{12}\right)\left(S^{23}(a, b, c)\right) & & \\
& =\left(S^{23} \circ S^{12} \circ S^{23}\right)(a, b, c) & &
\end{aligned}
$$

Then, $S(a, b)=(\neg a \longrightarrow b, \neg 1)$ is a solution to the set-theoretical Yang-Baxter equation in the W-algebra $A$.

Lemma 3. Let $\langle A, \longrightarrow, \neg, 1\rangle$ be a W-algebra. Then $S(a, b)=(\neg b, \neg a)$ is a solution to the set-theoretical Yang-Baxter equation.

Theorem 2. Let $\langle A, \longrightarrow, \neg, 1\rangle$ be a W-algebra. Then $S(a, b)=((a \longrightarrow b) \longrightarrow b, b)$ is a solution to the set-theoretical Yang-Baxter equation.

Proof. $S^{12}$ and $S^{23}$ are defined in the following forms:

$$
S^{12}(a, b, c)=((a \longrightarrow b) \longrightarrow b, b, c), \quad S^{23}(a, b, c)=(a,(b \longrightarrow c) \longrightarrow c, c) .
$$


For all $(a, b, c) \in A^{3}$, we have

$$
\begin{aligned}
& \left(S^{12} \circ S^{23} \circ S^{12}\right)(a, b, c)=S^{12}\left(S^{23}\left(S^{12}(a, b, c)\right)\right) \\
& =S^{12}\left(S^{23}((a \longrightarrow b) \longrightarrow b, b, c)\right) \\
& =S^{12}((a \longrightarrow b) \longrightarrow b,(b \longrightarrow c) \longrightarrow c, c) \\
& =((((a \longrightarrow b) \longrightarrow b) \longrightarrow \\
& ((b \longrightarrow c) \longrightarrow c)) \longrightarrow \\
& ((b \longrightarrow c) \longrightarrow c) \text {, } \\
& (b \longrightarrow c) \longrightarrow c, c) \\
& =((((a \longrightarrow b) \longrightarrow b) \longrightarrow \\
& ((c \longrightarrow b) \longrightarrow b)) \longrightarrow \\
& ((c \longrightarrow b) \longrightarrow b) \\
& (b \longrightarrow c) \longrightarrow c, c) \\
& =(((c \longrightarrow b) \longrightarrow(((a \longrightarrow b) \\
& \longrightarrow b) \longrightarrow b)) \longrightarrow((c \longrightarrow \\
& b) \longrightarrow b),(b \longrightarrow c) \longrightarrow c, c) \\
& =(((c \longrightarrow b) \longrightarrow((b \longrightarrow \\
& (a \longrightarrow b)) \longrightarrow(a \longrightarrow b))) \\
& \longrightarrow((c \longrightarrow b) \longrightarrow b) \text {, } \\
& (b \longrightarrow c) \longrightarrow c, c) \\
& =(((c \longrightarrow b) \longrightarrow(a \longrightarrow b)) \\
& \longrightarrow((c \longrightarrow b) \longrightarrow b) \text {, } \\
& (b \longrightarrow c) \longrightarrow c, c) \\
& =((a \longrightarrow((b \longrightarrow c) \longrightarrow c)) \\
& \longrightarrow((b \longrightarrow c) \longrightarrow c), \\
& (b \longrightarrow c) \longrightarrow c, c)
\end{aligned}
$$

and

$$
\begin{aligned}
\left(S^{23} \circ S^{12} \circ S^{23}\right)(a, b, c)= & S^{23}\left(S^{12}\left(S^{23}(a, b, c)\right)\right) \\
= & S^{23}\left(S^{12}(a,(b \longrightarrow c) \longrightarrow c, c)\right) \\
= & S^{23}((a \longrightarrow((b \longrightarrow c) \longrightarrow c)) \\
& \longrightarrow((b \longrightarrow c) \longrightarrow c) \\
= & ((a \longrightarrow c) \longrightarrow c, c) \\
& ((b \longrightarrow c) \longrightarrow c),(((b \longrightarrow c)) \longrightarrow \\
= & c) \longrightarrow c) \\
& ((a \longrightarrow((b \longrightarrow c) \longrightarrow c)) \\
& (b \longrightarrow c) \longrightarrow c) \longrightarrow c)
\end{aligned}
$$

Then, $S(a, b)=((a \longrightarrow b) \longrightarrow b, b)$ is a solution to the set-theoretical Yang-Baxter equation in the W-algebra $A$.

Corollary 1. Let $\langle A, \longrightarrow, \neg, 1\rangle$ be a W-algebra. Then $S(a, b)=((b \longrightarrow a) \longrightarrow a, b), S(a, b)=$ $((b \longrightarrow a) \longrightarrow a, a)$ and $S(a, b)=((a \longrightarrow b) \longrightarrow b, a)$ are solutions to the set-theoretical Yang-Baxter equation.

Proof. The proof is completed from (W3) and Theorem 2. 
Proposition 1. [23] Let $A$ be a $W$-algebra. The binary relation $\leq$ defined on $A$ as follows

$$
a \leq b \text { if and only if } a \longrightarrow b=1
$$

is a partial order on $A$.

Proposition 2. [23] Let $A$ be a $W$-algebra and $\leq$ be a partial order on $A$. If the join and the meet operators are defined by

$$
p \vee q=(p \longrightarrow q) \longrightarrow q
$$

and

$$
p \wedge q=\neg(p \longrightarrow \neg(p \longrightarrow q)),
$$

then this partial order determines a lattice on $A$.

Proposition 3. [23] Any W-algebra satisfies the following implications and equations

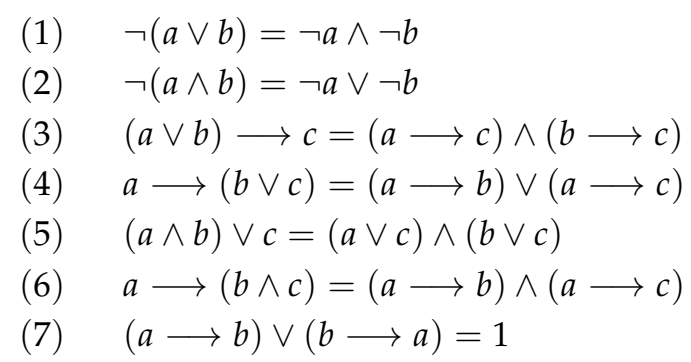

Lemma 4. Any W-algebra $A$ is a Boolean algebra if and only if $\neg a \longrightarrow a=$ a for all $a \in A$.

Proof. $(\Rightarrow)$ Assume that a W-algebra $A$ is a Boolean algebra. By using properties of Boolean algebras and proposition 2, we obtain

$$
1=\neg a \vee a=(\neg a \longrightarrow a) \longrightarrow a
$$

for all $a \in A$. Since we already have that $a=1 \longrightarrow a$ for all $a \in A$ from (W1), we get

$$
\begin{aligned}
a & =1 \longrightarrow a \\
& =((\neg a \longrightarrow a) \longrightarrow a) \longrightarrow a \\
& =(a \longrightarrow(\neg a \longrightarrow a)) \longrightarrow(\neg a \longrightarrow a) \\
& =1 \longrightarrow(\neg a \longrightarrow a) \\
& =(\neg a \longrightarrow a) .
\end{aligned}
$$

Consequently, $\neg a \longrightarrow a=a$ for all $a \in A$.

$(\Leftarrow)$ Suppose that $A$ is a W-algebra and let $\neg a \longrightarrow a=a$ for all $a \in A$. Since

$$
\begin{aligned}
\neg a \vee a & =((\neg a \longrightarrow a) \longrightarrow a) \\
& =a \longrightarrow a \\
& =1
\end{aligned}
$$

(Proposition 2)

and

$$
\begin{aligned}
\neg a \wedge a & =\neg(\neg a \longrightarrow \neg(\neg a \longrightarrow a)) \\
& =\neg(\neg a \longrightarrow \neg a) \\
& =\neg 1 \\
& =0,
\end{aligned}
$$


we have that the negation $\neg$ is a complementation, and the least element of $A$ is 0 defined as $\neg 1=0$ and 1 is the greatest element in $A$. Thus, $A$ is a bounded lattice with a complementation.

It remains to see that $A$ is distributive. By Proposition 3 (5), we already have that $(a \wedge b) \vee c=$ $(a \vee c) \wedge(b \vee c)$ for all $a, b, c \in A$. Besides, we obtain that

$$
\begin{aligned}
(a \vee b) \wedge c & =\neg((a \vee b) \longrightarrow \neg((a \vee b) \longrightarrow c)) & & \text { (Proposition 2) } \\
& =\neg((\neg c \longrightarrow \neg(a \vee b)) \longrightarrow \neg(a \vee b)) & & (\text { Lemma 2 (iii)-(iv)) } \\
& =\neg((\neg(a \vee b) \longrightarrow \neg c) \longrightarrow \neg c) & & (\text { W3 }) \\
& =\neg((c \longrightarrow(a \vee b)) \longrightarrow \neg c) & & (\text { Lemma 2 (iv)) } \\
& =\neg(((c \longrightarrow a) \vee(c \longrightarrow b)) \longrightarrow \neg c) & & \text { Proposition 3 (4)) } \\
& =\neg(((c \longrightarrow a) \longrightarrow \neg) \wedge((c \longrightarrow b) \longrightarrow \neg c)) & & \text { Proposition 3 (3)) } \\
& =\neg((c \longrightarrow a) \longrightarrow \neg c \vee \neg((c \longrightarrow b) \longrightarrow \neg) & & (\text { Proposition 3 (2)) } \\
& =\neg((\neg a \longrightarrow \neg) \longrightarrow \neg) \vee \neg((\neg b \longrightarrow \neg c) \longrightarrow \neg) & & \text { (Lemma 2 (iii)-(iv)) } \\
& =\neg(\neg a \vee \neg c) \vee \neg(\neg b \vee \neg c) & & \text { Proposition 2) } \\
& =(\neg \neg a \wedge \neg \neg c) \vee(\neg \neg b \wedge \neg \neg c) & & \text { (Proposition 3 (1)) } \\
& =(a \wedge c) \vee(b \wedge c) . & & \text { (Lemma 2 (iii)) }
\end{aligned}
$$

Hence, $A$ is a bounded distributive lattice with a complementation, that is, a W-algebra $A$ is a Boolean algebra.

The proof of the following theorem is given by F.F. Nichita.

Theorem 3. [9] Let $(A, \vee, \wedge, 0,1, \neg)$ be a Boolean algebra. Then $S(a, b)=(a \vee b, a \wedge b)$ is a solution to the set-theoretical Yang-Baxter equation.

Proof. Let $(A, \vee, \wedge, 0,1, \neg)$ be a Boolean algebra. $S^{12}$ and $S^{23}$ are defined in the following forms:

$$
S^{12}(a, b, c)=(a \vee b, a \wedge b, c), \quad S^{23}(a, b, c)=(a, b \vee c, b \wedge c) .
$$

For all $(a, b, c) \in A^{3}$, we obtain

$$
\begin{aligned}
\left(S^{12} \circ S^{23} \circ S^{12}\right)(a, b, c) & =S^{12}\left(S^{23}\left(S^{12}(a, b, c)\right)\right) \\
& =S^{12}\left(S^{23}(a \vee b, a \wedge b, c)\right) \\
& =S^{12}(a \vee b,(a \wedge b) \vee c,(a \wedge b) \wedge c) \\
& =((a \vee b) \vee((a \wedge b) \vee c),(a \vee b) \wedge((a \wedge b) \vee c),(a \wedge b) \wedge c) \\
& =(((a \vee b) \vee(a \wedge b)) \vee c,(a \vee b) \wedge((a \vee c) \wedge(b \vee c)),(a \wedge b) \wedge c)) \\
& =((a \vee(b \vee(a \wedge b))) \vee c,((a \vee b) \wedge(a \vee c)) \wedge(b \vee c), a \wedge(b \wedge c)) \\
& =((a \vee b) \vee c,(a \vee(b \wedge c)) \wedge(b \vee c), a \wedge(b \wedge c)) \\
& =(a \vee(b \vee c),(a \wedge(b \vee c)) \vee((b \wedge c) \wedge(b \vee c)), a \wedge(b \wedge c)) \\
& =(a \vee(b \vee c),(a \wedge(b \vee c)) \vee(b \wedge c), a \wedge(b \wedge c))
\end{aligned}
$$

and

$$
\begin{aligned}
\left(S^{23} \circ S^{12} \circ S^{23}\right)(a, b, c) & =S^{23}\left(S^{12}\left(S^{23}(a, b, c)\right)\right) \\
& =S^{23}\left(S^{12}(a, b \vee c, b \wedge c)\right) \\
& =S^{23}(a \vee(b \vee c), a \wedge(b \vee c), b \wedge c) \\
& =(a \vee(b \vee c),(a \wedge(b \vee c)) \vee(b \wedge c),(a \wedge(b \vee c)) \wedge(b \wedge c)) \\
& =(a \vee(b \vee c),(a \wedge(b \vee c)) \vee(b \wedge c), a \wedge((b \vee c) \wedge(b \wedge c))) \\
& =(a \vee(b \vee c),(a \wedge(b \vee c)) \vee(b \wedge c), a \wedge(b \wedge c)) .
\end{aligned}
$$

Then, $S(a, b)=(a \vee b, a \wedge b)$ is a solution to the set-theoretical Yang-Baxter equation.

Theorem 4. Let $(A, \longrightarrow, \neg, 1)$ be a W-algebra. If the identities 


$$
\neg((\neg a \longrightarrow b) \longrightarrow \neg c)=(a \longrightarrow \neg c) \longrightarrow \neg(b \longrightarrow \neg)
$$

or

$$
(a \longrightarrow \neg) \longrightarrow c=\neg((\neg a \longrightarrow c) \longrightarrow \neg(\neg b \longrightarrow c))
$$

are satisfied for all $a, b$ and $c$ in $A$, then

$$
S(a, b)=((a \longrightarrow b) \longrightarrow b, \neg(a \longrightarrow \neg(a \longrightarrow b)))
$$

and

$$
S(a, b)=(\neg a \longrightarrow b, \neg(a \longrightarrow \neg b))
$$

are solutions to the set-theoretical Yang-Baxter equation in the W-algebra $A$.

Proof. Substituting 1 instead of $a$ and $b$, or $\neg 1$ instead of $a$ and $b$ in identities (3) and (4), respectively, we obtain $c=\neg c \longrightarrow c$ or $\neg c=c \longrightarrow \neg$ by Lemma 2 (i) and (iii), and (W1). Then $A$ is a Boolean algebra by Lemma 4 . From the definitions of join and meet operators and Theorem 3 ,

$$
\begin{aligned}
S(a, b) & =((a \longrightarrow b) \longrightarrow b, \neg(a \longrightarrow \neg(a \longrightarrow b))) \\
& =(a \vee b, a \wedge b)
\end{aligned}
$$

is a solution to the set-theoretical Yang-Baxter equation in the $\mathrm{W}$-algebra $A$. Additionally, putting simultaneously $\neg b$ instead of $b$ and $b$ instead of $c$ in identity (4), we get

$$
(a \longrightarrow \neg \neg b) \longrightarrow b=\neg((\neg a \longrightarrow b) \longrightarrow \neg(\neg \neg b \longrightarrow b)) .
$$

Thus, we have

$$
\begin{aligned}
(a \longrightarrow b) \longrightarrow b & =(a \longrightarrow \neg \neg b) \longrightarrow b & & \text { Lemma 2 (iii)) } \\
& =\neg((\neg a \longrightarrow b) \longrightarrow \neg(b \longrightarrow b)) & & \left(*_{2}\right) \\
& =\neg((\neg a \longrightarrow b) \longrightarrow \neg 1) & & (\text { W5 }) \\
& =\neg \neg(\neg a \longrightarrow b) & & (\text { Lemma 2 (ii)) } \\
& =\neg a \longrightarrow b . & & (\text { Lemma 2 (iii)) }
\end{aligned}
$$

By applying Lemma 2 (iii)-(iv) to identity (3), we get

$$
\neg(c \longrightarrow \neg(\neg a \longrightarrow b))=(c \longrightarrow \neg a) \longrightarrow \neg(c \longrightarrow \neg) .
$$

Therefore, we obtain

$$
\begin{aligned}
\neg(a \longrightarrow \neg(a \longrightarrow b)) & =\neg(a \longrightarrow \neg(\neg \neg a \longrightarrow b)) \\
& =(a \longrightarrow \neg \neg a) \longrightarrow \neg(a \longrightarrow \neg b) \\
& =(a \longrightarrow a) \longrightarrow \neg(a \longrightarrow \neg b) \\
& =1 \longrightarrow \neg(a \longrightarrow \neg) \\
& =\neg(a \longrightarrow \neg b) .
\end{aligned}
$$

Hence, $S(a, b)=(\neg a \longrightarrow b, \neg(a \longrightarrow \neg b))$ is a solution to the set-theoretical Yang-Baxter equation in the $\mathrm{W}$-algebra $A$. 
Remark 1. Notice that the map $S(a, b)=(a \longrightarrow b, a)$ is a solution to the set-theoretical Yang-Baxter equation in Boolean algebras (Theorem 5.1 in [24]) while it is not a solution in Wajsberg algebras. Indeed, in W-algebras, we have

$$
\begin{aligned}
\left(S^{12} \circ S^{23} \circ S^{12}\right)(a, b, c) & =S^{12}\left(S^{23}\left(S^{12}(a, b, c)\right)\right) \\
& =S^{12}\left(S^{23}(a \longrightarrow b, a, c)\right) \\
& =S^{12}(a \longrightarrow b, a \longrightarrow c, a) \\
& =((a \longrightarrow b) \longrightarrow(a \longrightarrow c), a \longrightarrow b, a)
\end{aligned}
$$

and

$$
\begin{aligned}
\left(S^{23} \circ S^{12} \circ S^{23}\right)(a, b, c) & =S^{23}\left(S^{12}\left(S^{12}(a, b, c)\right)\right) \\
& =S^{23}\left(S^{12}(a, b \longrightarrow c, b)\right) \\
& =S^{23}((a \longrightarrow b) \longrightarrow c, a, b) \\
& =(a \longrightarrow(b \longrightarrow c), a \longrightarrow b, a) .
\end{aligned}
$$

Then, we get $\left(S^{12} \circ S^{23} \circ S^{12}\right)(a, b, c) \neq\left(S^{23} \circ S^{12} \circ S^{23}\right)(a, b, c)$ for all $a, b, c$.

However, under the condition that $a \longrightarrow(b \longrightarrow c)=(a \longrightarrow b) \longrightarrow(a \longrightarrow c)$ holds for any $a, b, c$, we have that $S(a, b)=(a \longrightarrow b, a)$ is a solution to the set-theoretical Yang-Baxter equation in W-algebras.

Lemma 5. The following equations hold in every W-algebra:

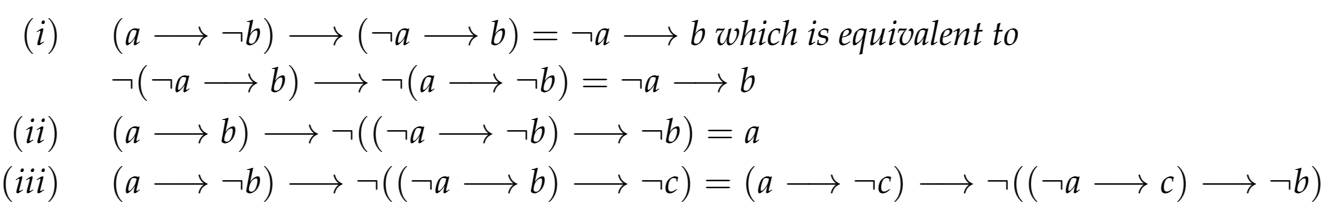

\section{Proof.}

(i)

$$
\begin{aligned}
(a \longrightarrow \neg b) \longrightarrow(\neg a \longrightarrow b)= & 1 \longrightarrow((a \longrightarrow \neg b) \longrightarrow(\neg a \longrightarrow b)) \\
= & ((\neg a \longrightarrow b) \longrightarrow((a \longrightarrow \neg b) \longrightarrow \\
& (\neg a \longrightarrow b))) \longrightarrow((a \longrightarrow \neg) \longrightarrow \\
& (\neg a \longrightarrow b)) \\
= & (((a \longrightarrow \neg b) \longrightarrow(\neg a \longrightarrow b)) \\
& \longrightarrow(\neg a \longrightarrow b)) \longrightarrow(\neg a \longrightarrow b) \\
= & ((a \longrightarrow \neg) \vee(\neg a \longrightarrow b)) \\
= & 1 \longrightarrow(\neg a \longrightarrow b) \\
= & \neg a \longrightarrow b \longrightarrow b)
\end{aligned}
$$

is equivalent to

$$
\neg(\neg a \longrightarrow b) \longrightarrow \neg(a \longrightarrow \neg b)=(a \longrightarrow \neg b) \longrightarrow(\neg a \longrightarrow b)=\neg a \longrightarrow b
$$

from Lemma 2 (iv). 
(ii)

$$
\begin{aligned}
(a \longrightarrow b) \longrightarrow \neg((\neg a \longrightarrow \neg b) \longrightarrow \neg b)= & (a \longrightarrow b) \longrightarrow \\
& \neg(\neg a \vee \neg b) \\
= & (a \longrightarrow b) \longrightarrow(a \wedge b) \\
= & ((a \longrightarrow b) \longrightarrow a) \wedge \\
& ((a \longrightarrow b) \longrightarrow b) \\
= & ((a \longrightarrow b) \longrightarrow a) \wedge \\
& ((b \longrightarrow a) \longrightarrow a) \\
= & ((a \longrightarrow b) \vee \\
& (b \longrightarrow a)) \longrightarrow a \\
= & 1 \longrightarrow a \\
= & a
\end{aligned}
$$

(iii) By Proposition $3(7)$, we already have that $(a \longrightarrow \neg b) \vee(\neg b \longrightarrow a)=1$ and $(a \longrightarrow \neg c) \vee$ $(\neg c \longrightarrow a)=1$. Thus, from definition of the join operator $\vee, a \longrightarrow \neg b=1$ or $\neg a \longrightarrow$ $b=\neg b \longrightarrow a=1$ (Lemma $2($ iii) $-($ iv)) and $a \longrightarrow \neg c=1$ or $\neg a \longrightarrow c=\neg c \longrightarrow a=$ 1 (Lemma $2($ iii $)-(i v))$.

Case 1. Assume that $a \longrightarrow \neg b=1$ and $a \longrightarrow \neg c=1$. Then by using $a \longrightarrow \neg b=1$, we get

$$
(a \longrightarrow \neg b) \longrightarrow \neg((\neg a \longrightarrow b) \longrightarrow \neg c) \quad=\neg((\neg a \longrightarrow b) \longrightarrow \neg c)
$$

and by using $a \longrightarrow \neg c=1$ we have

$$
(a \longrightarrow \neg c) \longrightarrow \neg((\neg a \longrightarrow c) \longrightarrow \neg b)=\neg((\neg a \longrightarrow c) \longrightarrow \neg b) .
$$

Case 2. Assume that $a \longrightarrow \neg b=1$ and $\neg a \longrightarrow c=1$. Then by using $a \longrightarrow \neg b=1$, we have

$$
(a \longrightarrow \neg b) \longrightarrow \neg((\neg a \longrightarrow b) \longrightarrow \neg c) \quad=\quad \neg((\neg a \longrightarrow b) \longrightarrow \neg c)
$$

and by using $\neg a \longrightarrow c=1$ we get

$$
(a \longrightarrow \neg c) \longrightarrow \neg((\neg a \longrightarrow c) \longrightarrow \neg b)=(a \longrightarrow \neg c) \longrightarrow b . \quad((W 1) \text { and Lemma } 2(\text { iii }))
$$

Case 3. Suppose that $\neg a \longrightarrow b=1$ and $a \longrightarrow \neg c=1$. Then by using $\neg a \longrightarrow b=1$, we get

$$
(a \longrightarrow \neg b) \longrightarrow \neg((\neg a \longrightarrow b) \longrightarrow \neg c)=(a \longrightarrow \neg b) \longrightarrow c \quad((W 1) \text { and Lemma } 2 \text { (iii) })
$$

and by using $a \longrightarrow \neg c=1$ we have

$$
(a \longrightarrow \neg c) \longrightarrow \neg((\neg a \longrightarrow c) \longrightarrow \neg b)=\neg((\neg a \longrightarrow c) \longrightarrow \neg b) .
$$

Case 4. Suppose that $\neg a \longrightarrow b=1$ and $\neg a \longrightarrow c=1$. Then by using $\neg a \longrightarrow b=1$, we get

$$
(a \longrightarrow \neg b) \longrightarrow \neg((\neg a \longrightarrow b) \longrightarrow \neg c)=(a \longrightarrow \neg b) \longrightarrow c \quad((\text { W1) and Lemma } 2 \text { (iii) })
$$

and by using $\neg a \longrightarrow c=1$ we have

$$
(a \longrightarrow \neg c) \longrightarrow \neg((\neg a \longrightarrow c) \longrightarrow \neg b)=(a \longrightarrow \neg c) \longrightarrow b . \quad((W 1) \text { and Lemma } 2 \text { (iii) })
$$


Hence, by Case 1, 2, 3 and 4,

$$
(a \longrightarrow \neg b) \longrightarrow \neg((\neg a \longrightarrow b) \longrightarrow \neg c)=(a \longrightarrow \neg c) \longrightarrow \neg((\neg a \longrightarrow c) \longrightarrow \neg b)
$$

holds in every W-algebra.

Proposition 4. The following identity

$$
(a \longrightarrow \neg b) \longrightarrow \neg((\neg a \longrightarrow b) \longrightarrow \neg c)=\neg((\neg a \longrightarrow b) \longrightarrow \neg((a \longrightarrow \neg b) \longrightarrow c))
$$

holds for every W-algebra.

Proof. By Proposition 3 (7), we already have that $(a \longrightarrow \neg b) \vee(\neg b \longrightarrow a)=1$, and from definition of the join operator $\vee, a \longrightarrow \neg b=1$ or $\neg a \longrightarrow b=\neg b \longrightarrow a=1$ (Lemma 2 (iii)-(iv)).

Assume that $a \longrightarrow \neg b=1$. Then, we get

$$
\begin{aligned}
(a \longrightarrow \neg b) \longrightarrow \neg((\neg a \longrightarrow b) \longrightarrow \neg c)= & 1 \longrightarrow \neg((\neg a \longrightarrow b) \longrightarrow \neg c) & & \text { (Hypothesis) } \\
= & \neg((\neg a \longrightarrow b) \longrightarrow \neg(1 \longrightarrow c)) & & \text { (W1) } \\
= & \neg((\neg a \longrightarrow b) \longrightarrow & & \\
& \neg((a \longrightarrow \neg) \longrightarrow c)) & & \text { (Hypothesis })
\end{aligned}
$$

for all $a, b, c$ in $A$.

Suppose that $\neg a \longrightarrow b=1$. Then, we obtain

$$
\begin{aligned}
(a \longrightarrow \neg b) \longrightarrow \neg((\neg a \longrightarrow b) \longrightarrow \neg c)= & (a \longrightarrow \neg b) \longrightarrow \neg(1 \longrightarrow \neg c) & & \text { (Hypothesis }) \\
= & \neg(1 \longrightarrow \neg((a \longrightarrow \neg b) \longrightarrow c)) & & ((\text { W1 and Lemma } 2 \text { (iii) }) \\
= & \neg((\neg a \longrightarrow b) \longrightarrow & & \\
& \neg((a \longrightarrow \neg) \longrightarrow c)) & & \text { (Hypothesis })
\end{aligned}
$$

for all $a, b, c$ in $A$.

Corollary 2. Let $(A, \longrightarrow, \neg, 1)$ be a W-algebra. Then $S(a, b)=(\neg a \longrightarrow b, \neg(a \longrightarrow \neg b))$ is a solution to the set-theoretical Yang-Baxter equation in the W-algebra $A$.

Proof. $S^{12}$ and $S^{23}$ are defined in the following forms:

$$
S^{12}(a, b, c)=(\neg a \longrightarrow b, \neg(a \longrightarrow \neg b), c), \quad S^{23}(a, b, c)=(a, \neg b \longrightarrow c, \neg(b \longrightarrow \neg c)) .
$$


For all $(a, b, c) \in A^{3}$, we get

$$
\begin{aligned}
& \left(S^{12} \circ S^{23} \circ S^{12}\right)(a, b, c)=S^{12}\left(S^{23}\left(S^{12}(a, b, c)\right)\right) \\
& =S^{12}\left(S^{23}(\neg a \longrightarrow b, \neg(a \longrightarrow \neg b), z)\right) \\
& =S^{12}(\neg a \longrightarrow b, \neg \neg(a \longrightarrow \neg b) \longrightarrow c, \neg(\neg(a \longrightarrow \neg b) \longrightarrow \neg c)) \\
& =(\neg(\neg a \longrightarrow b) \longrightarrow(\neg \neg(a \longrightarrow \neg b) \longrightarrow c) \text {, } \\
& \neg((\neg a \longrightarrow b) \longrightarrow \neg(\neg \neg(a \longrightarrow \neg b) \longrightarrow c)), \\
& \neg(\neg(a \longrightarrow \neg b) \longrightarrow \neg c)) \\
& =(\neg(\neg a \longrightarrow b) \longrightarrow((a \longrightarrow \neg b) \longrightarrow c) \text {, } \\
& \neg((\neg a \longrightarrow b) \longrightarrow \neg((a \longrightarrow \neg b) \longrightarrow c)), \\
& \neg(\neg(a \longrightarrow \neg b) \longrightarrow \neg c)) \\
& =((a \longrightarrow \neg b) \longrightarrow(\neg(\neg a \longrightarrow b) \longrightarrow c), \\
& \neg((\neg a \longrightarrow b) \longrightarrow \neg((a \longrightarrow \neg b) \longrightarrow c)), \\
& \neg(\neg(a \longrightarrow \neg b) \longrightarrow \neg c)) \\
& =((a \longrightarrow \neg b) \longrightarrow(\neg c \longrightarrow(\neg a \longrightarrow b)) \text {, } \\
& \neg((\neg a \longrightarrow b) \longrightarrow \neg((a \longrightarrow \neg b) \longrightarrow c)), \\
& \neg(c \longrightarrow(a \longrightarrow \neg b))) \\
& =(\neg c \longrightarrow((a \longrightarrow \neg b) \longrightarrow(\neg a \longrightarrow b)) \text {, } \\
& \neg((\neg a \longrightarrow b) \longrightarrow \neg((a \longrightarrow \neg b) \longrightarrow c)), \\
& \neg(c \longrightarrow(a \longrightarrow \neg b))) \\
& =(\neg c \longrightarrow(\neg a \longrightarrow b) \text {, } \\
& \neg((\neg a \longrightarrow b) \longrightarrow \neg((a \longrightarrow \neg b) \longrightarrow c)), \\
& \neg(c \longrightarrow(a \longrightarrow \neg b))) \\
& =(\neg a \longrightarrow(\neg c \longrightarrow b) \text {, } \\
& \neg((\neg a \longrightarrow b) \longrightarrow \neg((a \longrightarrow \neg b) \longrightarrow c)) \text {, } \\
& \neg(c \longrightarrow(a \longrightarrow \neg b))) \\
& =(\neg a \longrightarrow(\neg b \longrightarrow c) \text {, } \\
& \neg((\neg a \longrightarrow b) \longrightarrow \neg((a \longrightarrow \neg b) \longrightarrow c)), \\
& \neg(c \longrightarrow(a \longrightarrow \neg b))) \\
& =(\neg a \longrightarrow(\neg b \longrightarrow c) \text {, } \\
& (a \longrightarrow \neg b) \longrightarrow \neg((\neg a \longrightarrow b) \longrightarrow \neg c) \text {, } \\
& \neg(c \longrightarrow(a \longrightarrow \neg b)))
\end{aligned}
$$


and we have

$$
\begin{aligned}
& \left(S^{23} \circ S^{12} \circ S^{23}\right)(a, b, c)=S^{23}\left(S^{12}\left(S^{23}(a, b, c)\right)\right) \\
& =S^{23}\left(S^{12}(a, \neg b \longrightarrow c, \neg(b \longrightarrow \neg c))\right) \\
& =S^{23}(\neg a \longrightarrow(\neg b \longrightarrow c), \neg(a \longrightarrow \neg(\neg b \longrightarrow c)), \neg(b \longrightarrow \neg c)) \\
& =(\neg a \longrightarrow(\neg b \longrightarrow c) \text {, } \\
& \neg \neg(a \longrightarrow \neg(\neg b \longrightarrow c)) \longrightarrow \neg(b \longrightarrow \neg c), \\
& \neg(\neg(a \longrightarrow \neg(\neg b \longrightarrow c)) \longrightarrow \neg \neg(b \longrightarrow \neg))) \\
& =(\neg a \longrightarrow(\neg b \longrightarrow c) \text {, } \\
& (b \longrightarrow \neg c) \longrightarrow \neg(a \longrightarrow \neg(\neg b \longrightarrow c)), \\
& \neg(\neg(a \longrightarrow \neg(\neg b \longrightarrow c)) \longrightarrow \\
& (b \longrightarrow \neg c))) \\
& =(\neg a \longrightarrow(\neg b \longrightarrow c) \text {, } \\
& (b \longrightarrow \neg c) \longrightarrow \neg((\neg b \longrightarrow c) \longrightarrow \neg a), \\
& \neg(\neg(a \longrightarrow \neg(\neg b \longrightarrow c)) \longrightarrow \\
& (b \longrightarrow \neg c))) \\
& =(\neg a \longrightarrow(\neg b \longrightarrow c) \text {, } \\
& (b \longrightarrow \neg a) \longrightarrow \neg((\neg b \longrightarrow a) \longrightarrow \neg c), \\
& \neg(\neg(a \longrightarrow \neg(\neg b \longrightarrow c)) \longrightarrow \\
& (b \longrightarrow \neg c))) \\
& =(\neg a \longrightarrow(\neg b \longrightarrow c) \text {, } \\
& (a \longrightarrow \neg b) \longrightarrow \neg((\neg a \longrightarrow b) \longrightarrow \neg c), \\
& \neg(\neg(b \longrightarrow \neg c) \longrightarrow(a \longrightarrow \\
& \neg(\neg b \longrightarrow c)))) \\
& =(\neg a \longrightarrow(\neg b \longrightarrow c) \text {, } \\
& (a \longrightarrow \neg b) \longrightarrow \neg((\neg a \longrightarrow b) \longrightarrow \neg c) \text {, } \\
& \neg(a \longrightarrow(\neg(b \longrightarrow \neg c) \longrightarrow \\
& \neg(\neg b \longrightarrow c)))) \\
& =(\neg a \longrightarrow(\neg b \longrightarrow c) \text {, } \\
& (a \longrightarrow \neg b) \longrightarrow \neg((\neg a \longrightarrow b) \longrightarrow \neg c), \\
& \neg(a \longrightarrow(b \longrightarrow \neg c))) \\
& =(\neg a \longrightarrow(\neg b \longrightarrow c) \text {, } \\
& (a \longrightarrow \neg b) \longrightarrow \neg((\neg a \longrightarrow b) \longrightarrow \neg c) \text {, } \\
& \neg(a \longrightarrow(c \longrightarrow \neg b))) \\
& =(\neg a \longrightarrow(\neg b \longrightarrow c) \text {, } \\
& (a \longrightarrow \neg b) \longrightarrow \neg((\neg a \longrightarrow b) \longrightarrow \neg c), \\
& \neg(c \longrightarrow(a \longrightarrow \neg b))) \text {. } \\
& \text { (Lemma } 2(i v)) \\
& \text { (Lemma } 2(\text { iii) })-(i v)) \\
& \text { (Lemma } 5(\text { iii)) } \\
& \text { (Lemma } 2(i i i)-(i v)) \\
& \text { (Lemma } 2(i i i)-(i v))
\end{aligned}
$$

Then, $S(a, b)=(\neg a \longrightarrow b, \neg(a \longrightarrow \neg b))$ is a solution to the set-theoretical Yang-Baxter equation in the $\mathrm{W}$-algebra $A$.

Acknowledgments: The authors thank the academic editor for their valuable comments and suggestions and the anonymous referees for his/her remarks which helped them to improve the presentation of the paper.

Author Contributions: The coauthors, Tahsin Oner and Tugce Katican, wrote the paper jointly and contributed equally to this work.

Conflicts of Interest: The authors declare no conflict of interest.

\section{References}

1. Perk, J.H.H.; Au, Y.H. Yang-Baxter Equations. In Encyclopedia of Mathematical Physics; Françoise, J.-P., Naber, G.L., Tsou, S.T., Eds.; Elseiver: Oxford, UK, 2006; Volume 5, pp. 465-473.

2. Baxter, R.J. Exactly Solved Models in Statical Mechanics; Academy Press: London, UK, 1982.

3. Baxter, R.J. Partition Function of the Eight-Vertex Lattice Model. Ann. Phys. 1972, 70, 193-228. 
4. Yang, C.N. Some Exact Results for the Many-Body Problem in One Dimension with Repulsive Delta-Function Interaction. Phys. Rev. Lett. 1967, 19, 1312-1315.

5. Jimbio, M. Yang-Baxter Equation in Integrable Systems; Advanced Series in Mathematical Physics; World Scientific Publishing Co. Inc.: Singapore, 1990; Volume 10.

6. Jimbio, M. Introduction to the Yang-Baxter Equation. Int. J. Mod. Phys. 1989, 4, 3759.

7. Lu, J.-H.; Yan, M.; Zhu, Y.-C. On the Set-Theoretical Yang-Baxter Equation. Duke Math. J. 2000, 104, 1-18.

8. Nichita, F.F. Hopf algebras, Quantum Groups and Yang-Baxter Equations, 2014 (Special Issue). Available online: http://www.mdpi.com/journal/axioms/special_issue/hopf_algebras_2014 (accessed on 22 June 2017).

9. Nichita, F.F. On the set-theoretical Yang-Baxter Equation. Acta Univ. Apulensis Math. Inf. 2003, 5, 97-100.

10. Nichita, F.F. Yang-Baxter Equations, Computational Methods and Applications. Axioms 2015, 4, 423-435.

11. Oner, T.; Senturk, I.; Oner, G. An Independent Set of Axioms of MV-Algebras and Solutions of the Set-Theoretical Yang-Baxter Equation. Axioms 2017, 6, 17, doi:10.3390/axioms6030017.

12. Gateva-Ivanova, T. Noetherian Properties of Skew-Polynomial Rings with Binomial Relations. Trans. Am. Math. Soc. 1994, 343, 203-219.

13. Gateva-Ivanova, T. Skew Polynomial Rings with Binomial Relations. J. Algebra 1996, 185, 710-753.

14. Gateva-Ivanova, T.; Van den Bergh, M. Semigroups of I-type. J. Algebra 1998, 206, 97-112.

15. Tate, J.; Van den Bergh, M. Homological Properties of Sklyanin Algebras. Invent. Math. 1996, 124, 619-647.

16. Etingof, P.; Schedler, T.; Soloviev, A. Set-Theoretical Solutions to the Quantum Yang-Baxter Equation. Duke Math. J. 1999, 100, 169-209.

17. Etingof, P.; Gelaki, S. A Method of Construction of Finite-Dimensional Triangular Semisimple Hopf Algebras. Math. Res. Lett. 1998, 5, 551-561.

18. Veselov, A.P. Yang-Baxter Maps and Integral Dynamics. Phys. Lett. A. 2003, 314, $214-221$.

19. Etingof, P. Geometric Crystals and Set-Theoretical Solutions to the Quantum Yang-Baxter Equation. Comm. Algebra 2003, 31, 1961-1973.

20. Cignoli, R.L.O.; D’ottaviano, I.M.L.; Mundici, D. Algebraic Foundations of Many-Valued Reasoning; Kluwer Academic Publishers: Dordrech, The Netherlands, 2000.

21. Radfar, A.; Rezaei, A.; Sadei, A.B. Extensions of BCK-algebras. Cogent Math. 2016, doi:10.1080/23311835.2016.1265297.

22. Pałasiński, M. The variety of all commutative BCK-algebras is generated by its finite members as a quasivariety. Demonstr. Math. 2012, 45, 495-518.

23. Font, J.M.; Rodriguez, A.J.; Torrens, A. Wajsberg Algebras. Stochastica 1984, 8, 5-31.

24. Solomon, M.; Nichita, F.F. On Transcendental Numbers: New Results and a Little History. Mathematics 2015, submitted.

(C) 2018 by the authors. Licensee MDPI, Basel, Switzerland. This article is an open access article distributed under the terms and conditions of the Creative Commons Attribution (CC BY) license (http:/ / creativecommons.org/licenses/by/4.0/). 